\title{
Omega-3 fatty acids and acute neurological trauma: a perspective on clinical translation*
}

\author{
Stacy GLADMAN \\ Siew-Na LIM \\ Simon DYALL \\ Martin M. KNIGHT \\ John V. PRIESTLEY \\ Adina T. MICHAEL-TITUS \\ Centre for Neuroscience and Trauma, \\ Blizard Institute, \\ Barts and the London School of \\ Medicine and Dentistry, \\ Queen Mary University of London, \\ UK \\ $<$ a.t.michael-titus@qmul.ac.uk>
}

\begin{abstract}
Acute neurological trauma remains one of the clinical areas with the most significant unmet needs worldwide. In the central nervous system, acute trauma has two stages: the primary injury and the secondary injury. The former is irreversible, and is a direct consequence of the impact. In the aftermath of the injury, a complex series of processes exacerbate the injury and amplify tissue damage. Some of these processes are local, others involve a systemic response. It is these processes which ultimately determine the clinical outcome. The aim of the treatments is a) to confer neuroprotection and b) to promote neuroregeneration. The results reported so far with omega-3 fatty acids in animal models of neurotrauma suggest that these compounds have the potential to offer a novel therapeutic approach and target both protection and regeneration. They lead to increased neuronal and glial survival, they can limit the damaging neuroinflammation and they can also protect neurites. Long chain omega-3 fatty acids such as eicosapentaenoic acid and docosahexaenoic acid have a complex pharmacodynamics, which leads potentially to the activation of a multitude of targets, including voltage and ligand-gated ion channels, transcription factors and G-protein coupled receptors. They can produce tissue-specific metabolites which have intrinsic activity, either on the same or on different cellular targets. The apparent large therapeutic window of omega-3 fatty acids is an advantage in the context of trauma, with patients in an unstable state, with multiple injuries. The specific use of omega-3 fatty acids in spinal cord injury and peripheral nerve injury will be discussed, focusing on issues which need to be addressed in order to translate successfully to the clinic the efficacy reported in the initial proof of concept animal studies.
\end{abstract}

Key words: omega-3 fatty acids, docosahexaenoic acid, central nervous system injury, peripheral nervous system injury, neuroprotection, neuroregeneration

decade, accumulating evidence shows that omega- 3 fatty acids have significant therapeutic potential in CNS injury. The present overview summarizes progress on this topic, and the required developments towards clinical translation in CNS injury, with a focus on spinal cord trauma, and also reports the first evidence that supports beneficial effects of these compounds in PNS injury.

\section{Omega-3 fatty acids and central nervous system injury - steps towards translation}

Spinal cord injury $(\mathrm{SCl})$ is a catastrophic event which can result in permanent and major disability. The estimated cost of treating an individual for life can reach over $\$ 3$ million. In Europe, care costs are estimated at around $€ 4$ billion per year. Injury to the spinal cord arises as a consequence of many types of trauma; the initial trauma often leads to forces such as dislocation, distraction and compression being exerted onto the nervous tissue, that ultimately lead to irreversible injury and cell death at the point of impact.

In the aftermath of $\mathrm{SCl}$, a complex chain of reactions is triggered around the injury epicentre, which will ultimately determine the degree of functional impairment (Profyris et al., 2004; De Biase et al., 2005). Haemorrhage develops 2011 , cf. OCL 18-4 and 18-5.

To cite this article: Gladman S, Lim SN, Dyall S, Knight MM, Priestley JV, Michael-Titus AT. Omega-3 fatty acids and acute neurological trauma: a perspective on clinical translation. OCL 2011; 18(6): 317-323. doi : 10.1684/ocl.2011.0421 
early, leading to tissue oedema, accompanied by a disruption of the blood flow in the cord. Compression of the spinal cord leads to anoxia, that is proportional to the severity of the initial injury. Oedema develops at the injury epicentre and spreads rostrocaudally. Furthermore, injury also triggers a complex inflammatory reaction, which starts with local activation of the microglia, followed by infiltration of neutrophils, systemic macrophages and T-cells. This inflammatory reaction is complex, and may significantly enhance the primary damage, but there is also evidence that some elements of inflammation exert a protective role (Crutcher et al., 2006; Donnelly and Popovich, 2008). The two main therapeutic strategies in $\mathrm{SCl}$ are based on neuroprotection (early intervention to protect vulnerable tissue in the early phase of the initial trauma) and neuroregeneration (delayed intervention to promote repair) (Kwon et al., 2004).

Traumatic brain injury (TBI) is the leading cause of disability and mortality in those under 50 years old. It is generally the result of falls, motor accidents, sports and war injuries. The incidence of $\mathrm{TBI}$ is increasing worldwide and it is estimated that around 500 in every 100,000 individuals suffer from TBI annually in the US and in Europe. In a manner similar to $\mathrm{SCl}$, tensile stretch forces are also an important factor in the pathology of TBI.

Treatment of $\mathrm{TBI}$, like in $\mathrm{SCl}$, is focused on neuroprotection and neurorepair. In spite of the importance of this condition, there are at present no neuroprotective treatments that could be used to protect the patient in the aftermath of injury. They would be associated with huge personal benefits for patients and carers. Such treatments would also have very significant impact in terms of public health costs. As $\mathrm{TBI}$ and $\mathrm{SCl}$ share many elements of pathophysiology, certain neuroprotective treatments could be beneficial in both.

The exploration of treatments with neuroprotective properties has led to many promising results in animal models of injury, which attempt to reproduce the conditions of injury in humans. However, numerous clinical trials in $\mathrm{SCl}$ and TBI, focused on neuroprotection, have failed to lead to an effective treatment. Treatments tested so far include: corticosteroids, opiate anatgonists, calcium channel blockers (e.g. nimodipine), antioxidants and free rad- ical scavengers (e.g tirilazad) and glutamate NMDA receptor antagonists (Hawryluk et al., 2008). This is reminiscent of the stroke field in which over one hundred clinical trials for acute stroke have failed. The reasons for the failures of acute $\mathrm{SCl}$ trials may reside partly in the intrinsic limitations of some of the trials and their design (Hawryluk et al., 2008) but also in the decisions leading perhaps hastily from a promising preclinical observation to a clinical study.

The process of drug discovery in neurotrauma involves the use of in vitro and in vivo models. The former allow a detailed analysis of the response of the neurones and glial cells to injury, and the mechanisms underlying the beneficial efects of compounds. There is also a wide variety of in vivo animals of CNS injury, which attempt to mimic the human trauma. Unfortunately, drugs are sometimes tested in such models with very unrealistic time windows (many treatments lose their effects if delayed by a few hours) and in only one injury model or only one species. It is also sometimes difficult to achieve in humans the drug concentrations that are achieved in animals in order to obtain efficacy. Furthermore, the relative importance of rescuing a small amount of tissue in the area surrounding the lesion in a small rodent (rat, mouse) $v s$. the need to protect a much more substantial volume of tissue in humans is often overlooked.

As the efficacy and safety of new treatments for neurotrauma must be investigated in animal models before initiation of clinical trials, a large variety of animal species including dogs, cats, sheep, monkeys, rabbits, rats and mice have been used for the modelling of $\mathrm{SCl}$ and TBI. What has become clear over decades of failure in translation is that it is essential that a new treatment is validated in several injury models in the same species, and/or injury models in different species. Rats and mice are the most widely used animals and they differ in their reponse to injury of the CNS. Genetically modified mice are widely used to study pathological events and although their response to injury may not be closer to humans, they offer the additional advantage of facilitating the analysis in vivo of some of the mechanistic aspects of new treatments, using specific genetic manipulation. It is well-established that mice exhibit a very different response to $\mathrm{SCl}$, not only in terms of functional recovery, but also in their tissue changes and in particular their inflammatory reaction, compared to that seen in other mammals. For example, in a study by Sroga and colleagues, microglia/macrophages showed a peak activation at 7 days post-injury, similar to what is seen in rats, and subtle decreases in labelling over the next 2-5 weeks. In contrast, the onset and magnitude of lymphocytic infiltration were markedly different between rats and mice. Maximal T-cell accumulation occurred earlier but to a lesser extent in rats compared with mice. One distinct finding in mice was the presence of cell clusters that resembled lymphocytes but did not express lymphocyte-specific markers; these cells extended from blood vessels within the fibrotic tissue matrix, and their phenotype was characteristic of fibrocytes, which are involved in wound healing. These species-specific neuroinflammatory aspects may result in the formation of a distinct tissue environment at the site of $\mathrm{SCl}$, and may also account for differences in neurological outccome (Sroga et al., 2003). Mice do not exhibit the progressive necrosis and larger central dramatic cavitation of the cord that occurs in rats and other mammals, in which a rim of preserved white matter surrounds a fluid-filled cystic cavity after contusion/compression trauma. In contrast, the injured mouse spinal cord shows after injury dense fibrous connective tissue, and if present, there are only very small cavities (microcysts) at the lesion site. The connective tissue matrix at the lesion site decreases in size along its rostrocaudal axis over time, and the small cavities disappear at the chronic time points rather than enlarging, which is different to the trend seen in rats, in which cavitation increases over time.

In $\mathrm{SCl}$ research, two types of injury models are used: (i) models that aim to mimic as closely as possible the type of $\mathrm{SCl}$ that is observed clinically (i.e. injuries associated with contusion or compression forces), and (ii) models in which specific sections of the cord or tracts are lesioned, which are appropriate for the study of regeneration (i.e. hemisection and transection models).

Omega-3 polyunsaturated fatty acids (PUFA) were shown almost a decade ago to have significant neuroprotective potential (Blondeau et al., 2002) and in particular they appeared to protect 
acutely the cord after an episode of spinal cord ischaemia (Lang-Lazdunski et al., 2003). Following these observations with alpha-linolenic acid, the biosynthetic precursor of long chain omega-3 PUFA such as docosahexaenoic acid (DHA), studies in our laboratory have shown that the intravenous administration of a bolus of DHA 30 min after hemisection SCl, dramatically improved functional and histological outcome in rats (King et al., 2006). These results were subsequently confirmed in a model of compression $\mathrm{SCl}$ (Huang et al., 2007a). In this model, we also showed that the neuroprotective effect of the acute intravenous DHA bolus is further enhanced by combination with a sustained dietary DHA supplementation, in the weeks follwing injury (Huang et al., 2007b; Ward et al., 2010). This confirmation of efficacy strengthens the probability that this treatment will indeed show protection in human $\mathrm{SCl}$. However, in order to increase further the probability of successful translation to clinic, it is important to show efficacy in more than one species and/or model of $\mathrm{SCl}$ and ideally, later on to replicate the success of the treatment in more than one independent laboratory. Therefore, we recently performed a study on the effect of the acute DHA treatment in a mouse compression $\mathrm{SCl}$ model, using a similar paradigm as that used in the rat, which assesses the effect of a single early acute intravenous administration, associated or not with chronic DHA dietary supplementation in the period following injury (Lim et al., 2010).

Our observations confirmed the neuroprotective effect of a single bolus of $\mathrm{DHA}$ administered intravenously at the dose of $500 \mathrm{nmol} / \mathrm{kg} 30 \mathrm{~min}$ after injury after compression SCl. The treatment led to a significant increase in tissue protection, as reflected by a multitude of cellular markers. For example, DHA led to enhanced neuronal survival (figure 1), as well as an increase in oligodendrocyte survival. A marked reduction in the microglial response after injury was also seen (figure 2).

In contrast to our previous study in the rat (Huang et al., 2007b), the significant neuroprotective effect of the acute DHA injection was not markedly enhanced for all the markers studied, by a combination with chronic dietary DHA supplementation (400 mg/kg/day) over the period of 28 days after injury. It is not possible to conclude from this first set of data in the mouse whether the effect of the acute single DHA injection would be enhanced in mice if a longer period of DHA dietary supplementation and/or a different dose are used. Furthermore, raised dietary DHA levels alone for 4 weeks following compression of the spinal cord did not protect significantly against either the neurological deficit or the histological damage.

This confirmation adds further support to the hope of successful clinical translation of $\mathrm{DHA}$ in $\mathrm{SCl}$, as an early intervention which could be delivered by emergency teams. The critical time window for this acute bolus intervention appears to be the $2 \mathrm{~h}$ period following injury, which is achievable, both within a civilian and military context. Finally, more extensive studies in the semiacute and chronc period after injury are required in order to better understand the potential of long-chain omega-3 PUFA in this period. Interestingly, there is some evidence that the use of fish oil containing lipid emulsions (which contain DHA but also eicosapentaenoic acid (EPA)), may be of benefit in critically ill patients with multiple trauma (Heller et al., 2006). However, it is likely that the mechanisms triggered by the acute bolus and the sustained exposure to DHA (diet or infusion) are quite different.

\section{Mechanism of action of omega-3 PUFA}

After $\mathrm{SCl}$, the primary injury area is compromised rapidly, but the injury also spreads. Metabolic and biochemi-

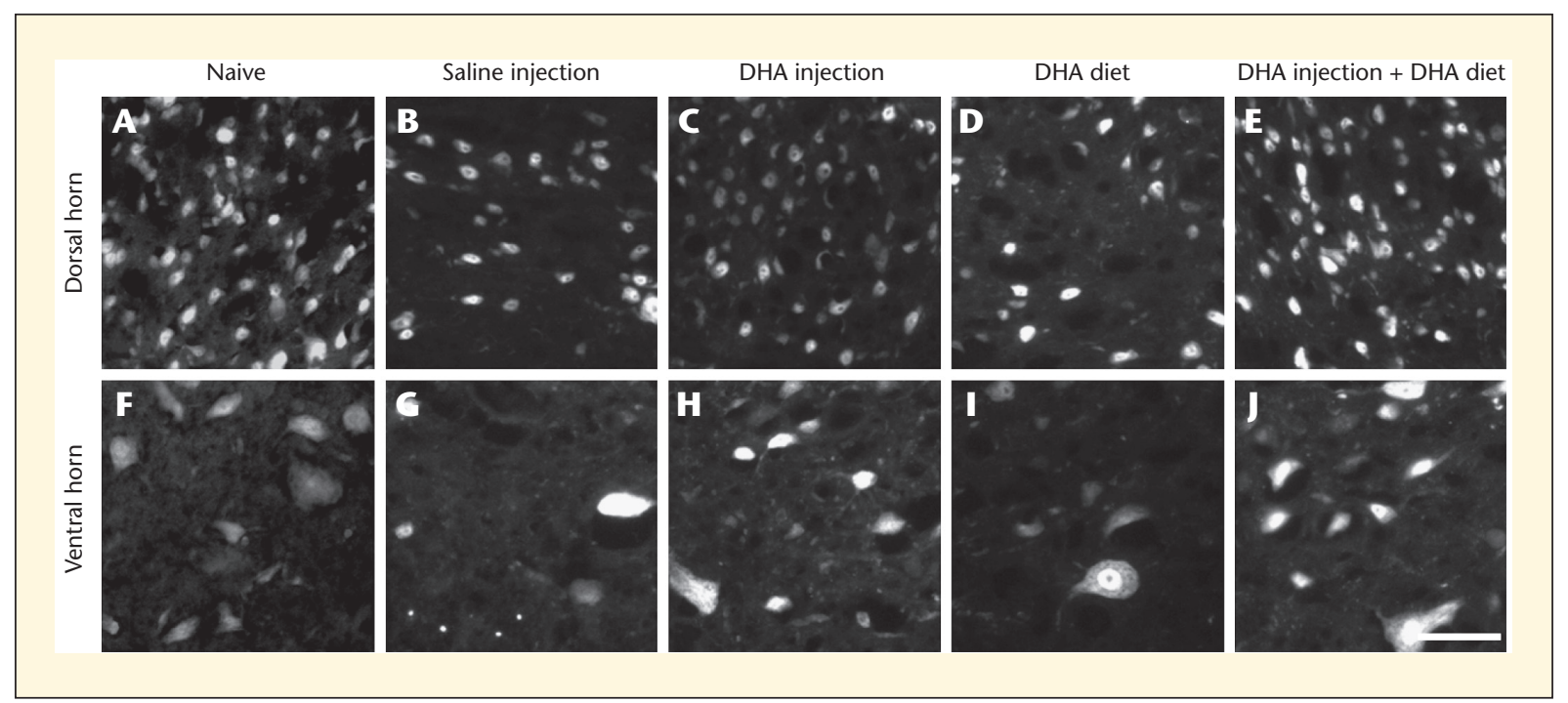

Figure 1. Neuronal protection in the dorsal horn and ventral horn of the spinal cord in mice injected with saline or DHA and then fed a DHA enriched diet or control diet. Representative images taken from the injury epicentre show that the number of NeuN-labelled cells in the animals treated with saline injection ( $B$ and $G$ ) and DHA diet alone ( $D$ and $I)$ was substantially less than naive control animals (A and $F)$. Animals receiving a DHA injection alone (C and $\mathrm{H}$ ) and DHA injection plus DHA diet (E and J) had more NeuN-labelled cells compared with saline-treated animals. Scale bar $=50 \mu \mathrm{m}$. (Lim et al., 2010). 


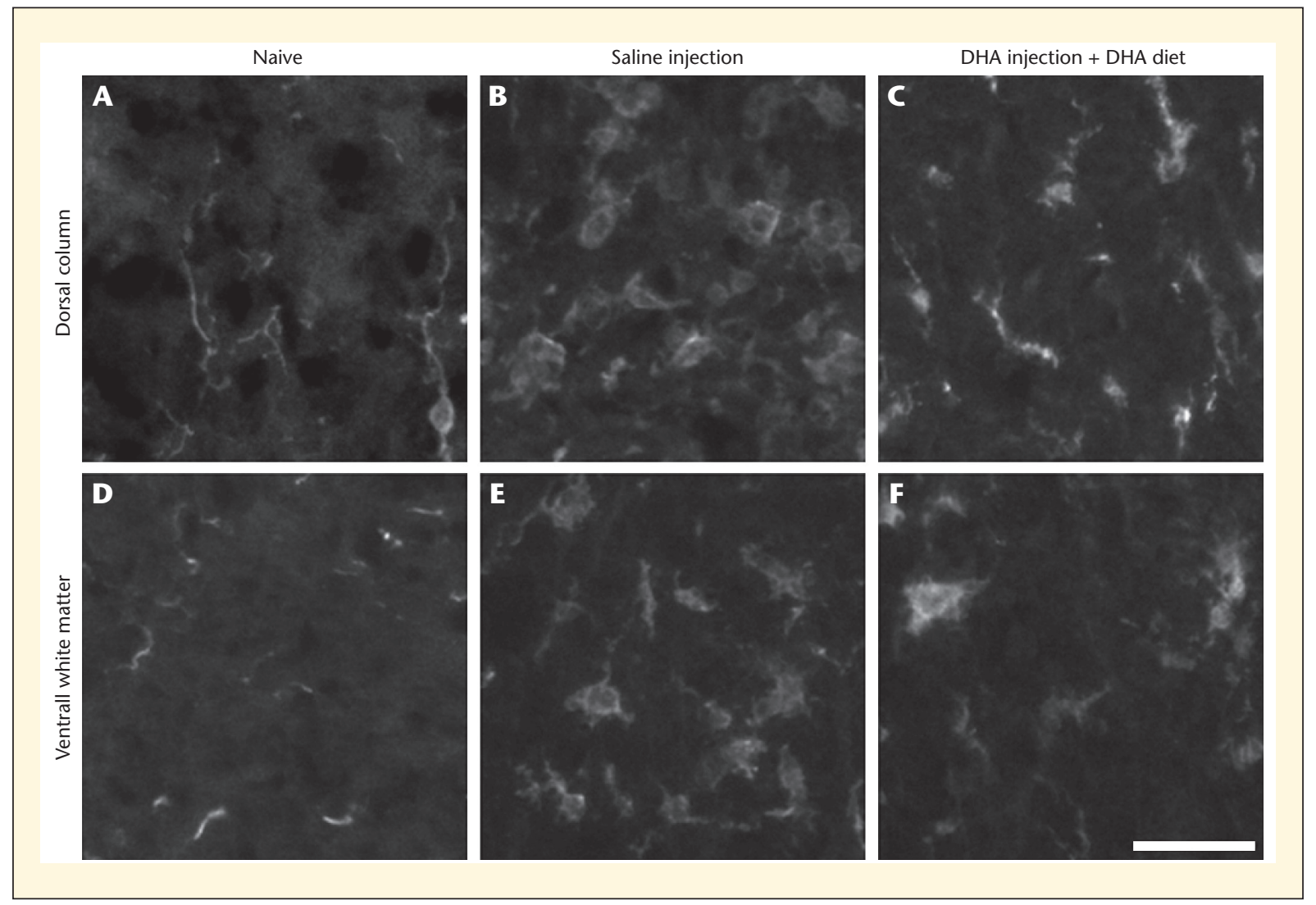

Figure 2. Decreased microglial activation in mice treated with saline injection and DHA injection plus DHA-enriched diet after spinal cord injury. Representative images taken from the epicentre show that resting microglial cells with ramified thin processes were noted in the naive tissue (A and $D)$. The microglia shape in saline-treated injured controls was larger and irregular ( $B$ and $E)$, reflecting activation. At 28 days after surgery, there appeared to be less Iba1 immunoreactivity in DHA-treated animals (C and F) compared with saline-treated animals (B and E). The effect is more marked in the dorsal horn compared to the ventral horn. Scale bar $=50 \mu \mathrm{m}$. (Lim et al., 2010).

cal changes, over a period of hours to days, increase the area of cell death. Excitotoxicity and increased oxidation are key neurochemical processe involved in injury (Hall and Braughler, 1986). Some of the cellular changes that develop in time also attempt to create a boundary between healthy and damaged tissue, and remove necrotic tissue. These changes include the appearance of reactive astrocytes, the activation of microglial cells and the infiltration of immune cells from the periphery. In tracts affected by the injury, Wallerian degeneration begins in the first days following injury and continues over a period of weeks. Gene profiling studies have identified hundreds of genes whose expression is either upregulated or downregulated at various time points after SCl (De Biase et al., 2005). The aim of neuroprotective treatments is to rescue CNS tissue that is under threat from the rapidly spreading damage. However, the extreme complexity of the reactions triggered by neurotrauma presents a tremendous challenge, to identify those events that are key to the evolving pathology and which could be critically targetted by DHA.

Omega-3 PUFAs are essential structural compounds in the CNS, but they also act as endogenous ligands at a variety of receptors and ion channels, and as substrates of enzymes. Their activity at potassium and sodium channels could be a major factor controlling hyperexcitability after injury (Vreugdenhil et al., 1996; Heurteaux et al., 2006). Continuous exposure to DHA can lead to significant changes in the biophysical properties of membranes. Increasing endogenous omega-3 PUFA levels through a raised DHA dietary level produces widespread effects on gene expression. One target mediating the effects on gene expression are nuclear receptors transcription factors. DHA acts as a ligand for the retinoid $X$ receptor (RXR) (Mata de Urquiza et al., 2000). RXR can heterodimerize with retinoic acid receptors (RAR) and act as a modulator of gene expression at retinoid-responsive promoters. Omega3 PUFAs can also activate PPARs (peroxisome proliferator activated receptors). PPARs can bind DNA as a heterodimer with RXR, and have been shown to have a therapeutic value as a target in $\mathrm{SCl}$ (McTigue et al., 2007).

It is important to note that following trauma, polyunsaturated fatty acids such as DHA are cleaved from membrane phospholipids to free (unesterified) DHA by phospholipase $A_{2}$ enzymes and the free DHA can then be converted to neuroactive metabolites such as neuroprotectin D1 (NPD1). Omega-3 PUFAs 


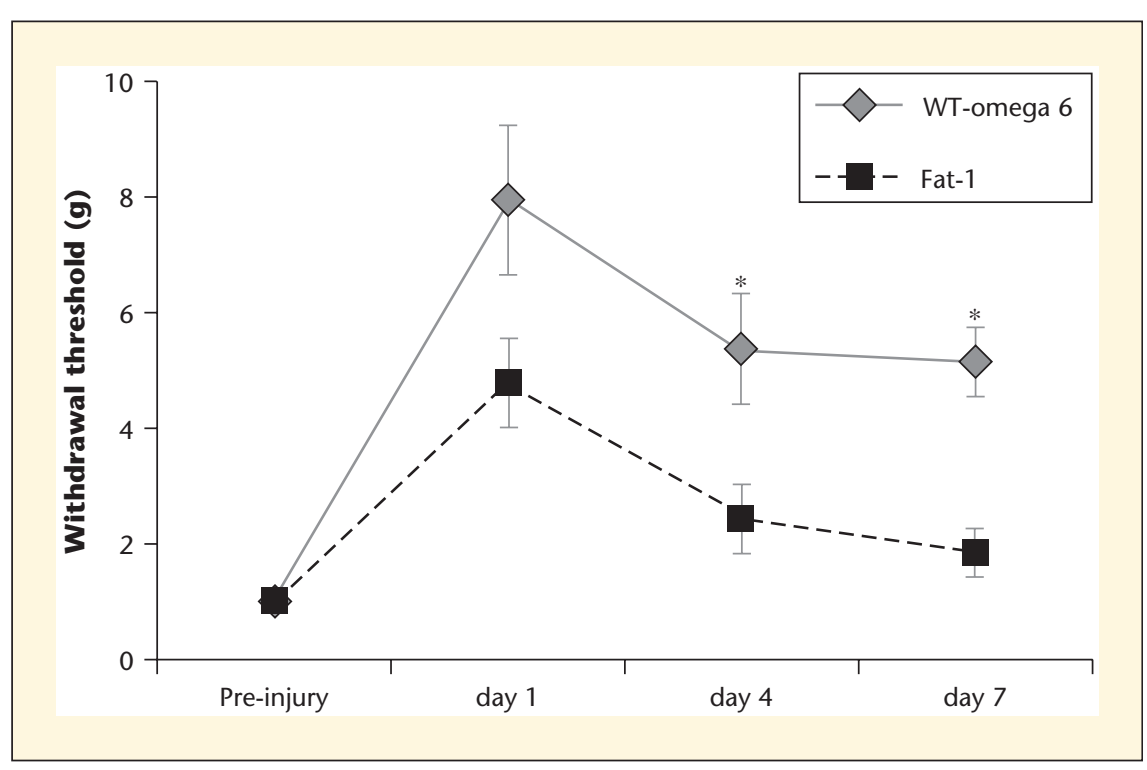

Figure 3. Recovery after peripheral nerve injury in wild type (WT) and fat- 1 mice. Animals were submitted to an injury of the sciatic nerve, and were tested functionally for 7 days. There was no difference in pre-injury withdrawal thresholds between WT-omega-6 and fat-1 groups, but at 4 days post-injury there was a statistical difference between the response of the two groups, and this continued to day $7\left({ }^{*} p<0.05\right)$. Error bars indicate S.E.M for $n=5$ animals/group. (Gladman et al., in press)

and their metabolites can up-regulate the expression of anti-apoptotic proteins such as the Bcl-2 family, whilst downregulating the apoptotic proteases caspase- 3 and 9, and pro-apoptotic signalling proteins including Bax, Bad, Bid and Bik. It has also been suggested that omega-3 PUFAs enhance the expression of neurotrophins, including brain-derived neurotrophic factor (BDNF). An increase in dietary omega-3 PUFAs resulted in BDNF levels being restored after experimental TBI in rats (Wu et al., 2004).

\section{Omega-3 fatty acids and their potetial in the management of peripheral nerve injury}

Peripheral nerve injury (PNI) occurs largely as a result of either direct mechanical trauma, disease, (such as diabetes), or toxicity associated with certain drugs. The various types of mechanical trauma include crush and compression injury, transection injury, and stretch injury. The latter could follow displacement of fractures and dislocation of joints. Unlike axons in the CNS, axons in the adult peripheral release mitogens for Schwann cells and fibroblasts, and cytokines that stimulate the synthesis of growth factors.

Many key events in the pathology of PNI reproduce events occurring in $\mathrm{CNS}$ trauma. These include : 1) production of free radicals which results in lipid peroxidation and oxidation of proteins and nucleic acids, 2) activation of proapoptotic proteases, 3) mitochondrial dysfunction, 4) activation of calpains leading to damage of the cytoskeleton, 5) activation of phospholipase $\mathrm{A}_{2}$ enzymes which release fatty acids such as arachidonic acid, triggering a local increase in deleterious prostaglandins and leukotrienes.

In humans, the distance over which a nerve must regenerate can be quite large, and for example it can take approximately 800 days for a nerve to regenerate from the shoulder, after a brachial plexus injury, to the hand. After such a time there will be irreversible damage to the denervated target organs, and full functional recovery will be unlikely. Therefore, therapies that can not only offer neuroprotection in the aftermath of PNI but also accelerate the rate of regeneration would be very beneficial in the clinic.

nervous system (PNS) can regenerate when damaged. Partial or complete axonal regeneration is essential for the functional recovery of nerves after injury. Satisfactory recovery usually occurs only in minor nerve injuries, or when the distance over which regeneration must occur is small (Jaquet et al., 2001). After a $\mathrm{PNI}$, rehabilitation can lead to some recovery. However, some patients may remain extensively incapacitated and are unable to return successfuly to an active life (Rosberg et al., 2005). Hence there is a need for new therapies that protect injured peripheral neurons and enhance regeneration, thus improving functional outcome.

Vascular changes accompany the neural changes seen after PNI, and this can exacerbate hypoxia and ischaemia. There is also an inflammatory reaction following PNI, largely believed to be beneficial for recovery. Macrophages are involved in phagocytosis of degenerating nerve fibres, which is a critical step enabling regeneration, as myelin contains many growth-inhibitory molecules, such as myelin-associated glycoprotein and oligodendrocyte myelin glycoprotein, as well as the Nogo receptor and the p75 neurotrophin receptor. Macrophages
The encouraging effects seen with long chain omega-3 PUFA suh as DHA in $\mathrm{SCl}$, have led us to explore the effects of omega-3 fatty acids in PNI. The aim of our first study was to assess the effect of increasing tissue levels of omega-3 PUFA on the response to a $\mathrm{PNI}$, sciatic nerve crush, in the mouse (Gladman et al., in press). Such an increase can be achieved through dietary supplementation, or alternatively through the use of the recently developed fat 1 mouse. These mice express the fat- 1 gene from $C$. elegans, which encodes a fatty acid desaturase not normally present in mammals. This enzyme can convert omega-6 into omega-3 PUFAs, leading to enrichment in tissue omega-3 PUFA levels. This genetic manipulation allows us to produce two different tissue fatty acid profiles (i.e. high vs. low omega-6/ omega-3 ratio), in wild type animals maintained on a diet enriched in omega-6 PUFA (WT-omega-6) vs. the fat-1 mice maintained on the same diet.

In agreement with previously published findings using an in vitro analysis of the response of primary sensory neurones to fatty acids (Robson et al., 2010) the 


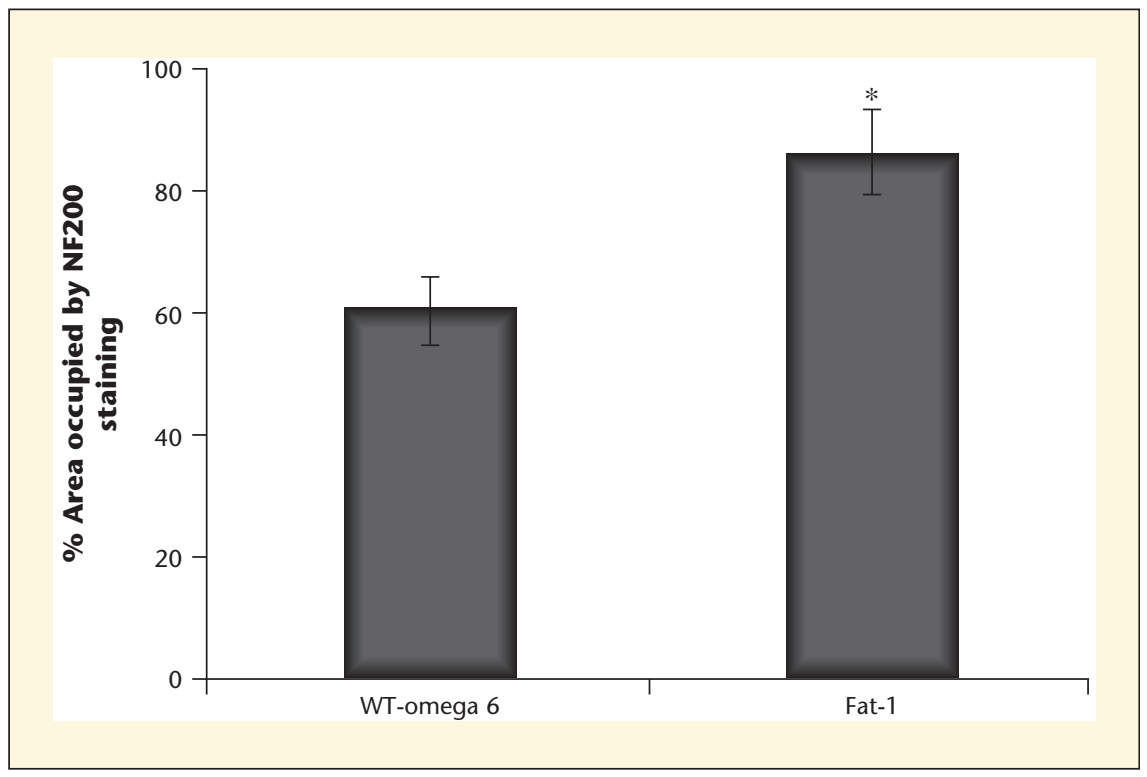

Figure 4. Neurofilament NF200 staining following sciatic nerve injury: Immunoreactivity for NF200 was analysed in transverse sections of the mouse sciatic nerve, $6 \mathrm{~mm}$ from the crush site 7 days after injury. NF200 staining shows that there was significantly less staining in the WTomega 6 group ( ${ }^{*} p<0.05$, means \pm S.E.M., $n=11$ animals/group). (Gladman et al., in press).

results of the study with fat- 1 mice demonstrate the intrinsic neurotrophic properties of omega-3 PUFAs. Thus, in vitro, dorsal root ganglia primary sensory neurones from fat-1 mice showed much more complex neurite outgrowth compared to wild type animals. The response to $\mathrm{PNI}$ in mice expressing the fat-1 gene was then examined using the sciatic nerve crush model. Behavioural observations showed that higher endogenous omega-3 PUFAs had a positive effect on the rate of sensory functional recovery. The sciatic functional index reflects locomotion and combines the coordination of motor and sensory reflexes. At 7 days postinjury there was a small but significant difference, with fat-1 mice regaining comparatively more function, thus indicative of an increased rate of recovery. We used the von Frey test to assess the motor response to a sensory stimulus and as expected, both groups lost initially sensation after the injury. By 4 days post-crush, the withdrawal threshold for fat- 1 mice was significantly lower than for WT-omega-6 mice (figure 3). This trend continued up to day 7 when the experiments were completed, thus suggesting that omega-3 PUFAs increase the rate of regeneration. However, by 1 day post-injury there was already a small difference in the force ful metabolites which have distinct receptors and associated signalling pathways (Serhan et al., 2004) or do they have a unique therapeutic value without conversion to metabolites? How will omega-3 PUFA, which have powerful efects on systemic inflammation (Calder, 2003; Mori and Beilin, 2004), modulate complex tissue reactions such as neuroinflammation, which has a dual function after neurotrauma (Donnelly and Popovich, 2008; Kigerl et al., 2009)? Complex questions which are awaiting the answers that will make safe clinical translation possible.

\section{REFERENCES}

Blondeau N, Widmann C, Lazdunski M, et al. Polyunsaturated fatty acids induce ischemic and epileptic tolerance. Neurosci 2002; 109: 231-41.

Calder PC. N-3 polyunsaturated fatty acids and inflammation: from molecular biology to the clinic. Lipids 2003; 38: 343-52.

Crutcher Ka, Gendelman He, Kipnis J, et al. Debate: "is increasing neuroinflammation beneficial for neural repair?". I Neuroimmune Pharmacol 2006; 1: 195-211.

to induce a response betwee the two groups. This is a strong indication that the injury was less severe in the fat-1 mice, likely due to the neuroprotective properties of omega-3 PUFA leading to more spared axons, and this could be the explanation for the observed improvements in both the von Frey test and the sciatic functional index. Additionally it could be hypothesised that omega-3 PUFAs led to an increase in collateral sprouting from spared axons in the sciatic nerve, and that this could further contribute to the increased rate of recovery seen in mice expressing the fat-1 gene. The fat-1 background also led to an increased staining for neurofilament, as a marker of axonal integrity (figure 4).

\section{Conclusion}

Omega-3 PUFA continue to represent a significant promise in CNS trauma, where progress towards clinical translation is being made, and there is also an emerging hope in the use of these compounds in treatment regimes for PNI. Many questions remain: what are the main mechanisms driving neuroprotection and possibly neuroregeneration with these compunds? Are the long-chain omega-3 PUFA acting only as pro-drugs, and precursors to power-
De Biase A, Knoblach SM, Di Giovanni S, et al. Gene expression profiling of experimental traumatic spinal cord injury as a function of distance from impact site and injury severity. Physiol Genomics 2005; 22: 368-81.

Donnelly DJ, Popovich PG. Inflammation and its role in neuroprotection, axonal regeneration and functional recovery after spinal cord injury. Exp Neurol 2008; 209: 378-88.

Gladman S, Huang W, Lim S, et al. Improved outcome after peripheral nerve injury in mice with increased levels of endogenous omega3 polyunsaturated fatty acids. I Neurosci, in press.

Hall ED, Braughler JM. Role of lipid peroxidation in post-traumatic spinal cord degeneration: a review. Cent Nerv Syst Trauma 1986; 3: 281-94.

Hawryluk GW, Rowland J, Kwon BK, et al. Protection and repair of the injured spinal cord: a review of completed, ongoing, and planned clinical trials for acute spinal cord injury. Neurosurg Focus 2008; 25: E14.

Heller AR, Rossler S, Litz RJ, et al. Omega-3 fatty acids improve the diagnosis-related clinical outcome. Crit Care Med 2006; 34: 972-9.

Heurteaux C, Laigle C, Blondeau N, et al. Alpha-linolenic acid and riluzole treatment confer cerebral protection and improve survival after focal brain ischemia. Neurosci 2006; 137: 241-51. 
Huang W, Bhavsar A, Ward RE, et al. Arachidonyl trifluoromethyl ketone is neuroprotective after spinal cord injury. I Neurotrauma 2009; 26: 1429-34.

Huang WL, George KJ, Ibba V, et al. The characteristics of neuronal injury in a static compression model of spinal cord injury in adult rats. Eur J Neurosci 2007a; 25: 362-72.

Huang WL, King VR, Curran OE, et al. A combination of intravenous and dietary docosahexaenoic acid significantly improves outcome after spinal cord injury. Brain 2007b ; 130: 3004-19.

Jaquet JB, Luijsterburg AJ, Kalmijn S, Kuypers PD, Hofman A, Hovius SE. Median, ulnar, and combined median-ulnar nerve injuries: functional outcome and return to productivity. I Trauma 2001; 51: 687-92.

Kigerl KA, Gensel JC, Ankeny DP, et al. Identification of two distinct macrophage subsets with divergent effects causing either neurotoxicity or regeneration in the injured mouse spinal cord. / Neurosci 2009; 29: 13435-44.

King VR, Huang WL, Dyall SC, et al. Omega-3 fatty acids improve recovery, whereas omega6 fatty acids worsen outcome, after spinal cord injury in the adult rat. I Neurosci 2006; 26: 4672-80.

Kwon BK, Tetzlaff W, Grauer JN, et al. Pathophysiology and pharmacologic treatment of acute spinal cord injury. Spine J 2004; 4: 451-64.
Lang-Lazdunski L, Blondeau N, Jarretou G, et $a l$. Linolenic acid prevents neuronal cell death and paraplegia after transient spinal cord ischemia in rats. J Vasc Surg 2003; 38: 564-75.

Lauritzen I, Blondeau N, Heurteaux C, et al. Polyunsaturated fatty acids are potent neuroprotectors. EMBO / 2000; 19: 1784-93.

Lim S, Huang W, Hall JCE, Priestley JV, Michael-Titus AT. Docosahexaenoic Acid is Neuroprotective After Spinal Cord Compression Injury in the Mouse. FENS meeting, Amsterdam, July 2010.

Mata de Urquiza A, Liu S, Sjoberg M, et al. Docosahexaenoic acid, a ligand for the retinoid $X$ receptor in mouse brain. Science 2000; 290: 2140-4.

McTigue DM, Tripathi R, Wei $\mathrm{P}$, et al. The PPAR gamma agonist Pioglitazone improves anatomical and locomotor recovery after rodent spinal cord injury. Exp Neurol 2007; 205: 396-406.

Mori Ta And Beilin LJ. Omega-3 fatty acids and inflammation. Curr Atheroscler Rep 2004; 6: 461-7.

Profyris C, Cheema SS, Zang D, et al. Degenerative and regenerative mechanisms governing spinal cord injury. Neurobiol Dis 2004; 15: 415-36.

Robson LG, Dyall S, Sidloff D, Michael-Titus AT. Omega-3 polyunsaturated fatty acids increase the neurite outgrowth of rat sensory neurones throughout development and in aged animals. Neurobiol Aging 2010; 31: 678-87.

Rosberg HE, Carlsson KS, Hojgard S, Lindgren B, Lundborg G, Dahlin LB. Injury to the human median and ulnar nerves in the forearm-analysis of costs for treatment and rehabilitation of 69 patients in southern Sweden. I Hand Surg Br 2005; 30: 35-39.

Serhan CN, Arita M, Hong S, et al. Resolvins, docosatrienes, and neuroprotectins, novel omega-3-derived mediators, and their endogenous aspirin-triggered epimers. Lipids 2004; 39: 1125-32.

Sroga JM, Jones TB, Kigerl KA, et al. Rats and mice exhibit distinct inflammatory reactions after spinal cord injury. I Comp Neurol 2003; 462: 223-240.

Vreugdenhil M, Bruehl C, Voskuyl RA, et al. Polyunsaturated fatty acids modulate sodium and calcium currents in CA1 neurons. Proc Natl Acad Sci U S A 1996; 93: 1255963.

Ward RE, Huang W, Curran OE, Priestley JV, Michael-Titus AT. Docosahexaenoic acid prevents white matter damage after spinal cord injury. I Neurotrauma 2010; 27: 1769-80.

Wu A, Ying Z, Gomez-Pinilla F. Dietary omega-3 fatty acids normalize BDNF levels, reduce oxidative damage, and counteract learning disability after traumatic brain injury in rats. I Neurotrauma 2004; 21: 1457-67. 